\title{
Wnt5a/ROR1 activates DAAM1 and promotes the migration in osteosarcoma cells
}

\author{
BIN DAI $^{1^{*}}$, YUCHENG SHEN $^{1 *}$, TING YAN $^{2}$ and AILIANG ZHANG ${ }^{3}$ \\ ${ }^{1}$ Department of Orthopedics, Binhai County People's Hospital, Binhai, Jiangsu 224500; \\ ${ }^{2}$ Safety Assessment and Research Center for Drug, Pesticide and Veterinary Drug of Jiangsu Province, \\ Nanjing Medical University, Nanjing, Jiangsu 211166; ${ }^{3}$ Department of Spine Surgery, \\ Third Affiliated Hospital of Soochow University, Changzhou, Jiangsu 213003, P.R. China
}

Received May 11, 2019; Accepted November 8, 2019

DOI: $10.3892 /$ or.2019.7424

\begin{abstract}
Receptor tyrosine kinase like orphan receptor 2 (ROR2) regulates Wnt5a-induced cell migration by phosphorylating PI3K/Akt and activating RhoA in osteosarcoma. However, the role of Wnt5a signaling and its corresponding receptors in the regulation of osteosarcoma metastasis remains poorly understood. ROR1 monoclonal antibody (mAb) and short hairpin (sh)RNA targeting ROR2 markedly inhibited the activity of dishevelled associated activator of morphogenesis 1 (DAAM1) and RhoA and retarded cell migration in osteosarcoma. ROR1 $\mathrm{mAb}$ and ROR2 shRNA destroyed the microfilament formation of osteosarcoma cells. Silencing of DAAM1 (with DAAM1 shRNA) downregulated RhoA activity and inhibited cell migration. The decrease of cell migration caused by DAAM1 shRNA was rescued by wild-type DAAM1 overexpression. DAAM1 and PI3K $\alpha /$ Akt were parallel signaling pathways mediating osteosarcoma cell migration in response to Wnt5a. It was concluded that Wnt5a promotes osteosarcoma cell migration via ROR1/2 receptors, and then activates DAAM1 and RhoA.
\end{abstract}

\section{Introduction}

Osteosarcoma is a primary cancerous tumor in bones and has an incidence rate of 3.4/1,000,000 per year worldwide $(1,2)$. In

Correspondence to: Dr Ailiang Zhang, Department of Spine Surgery, Third Affiliated Hospital of Soochow University, 185 Juqian Road, Changzhou, Jiangsu 213003, P.R. China

E-mail: ailiangzhang07@163.com

*Contributed equally

Abbreviations: ROR1, receptor tyrosine kinase-like orphan receptor 1; ROR2, receptor tyrosine kinase-like orphan receptor 2; DAAM1, disheveled-associated activator of morphogenesis 1; LRP, lipoprotein receptor-related protein; $\mathrm{mAb}$, monoclonal antibody; shRNA, short hairpin RNA

Key words: osteosarcoma, migration, Wnt5a, ROR1, DAAM1 the last 100 years, the 5-year survival rate of classical osteosarcoma has been $20 \%$ (2). Surgical and/or en bloc resection and radiotherapy are the primary options for the treatment of osteosarcoma. However, patients with advanced osteosarcoma often suffer from metastasis or other complications. The underlying molecular mechanism of cell migration is still unclear in osteosarcoma.

Wnt ligands are a group of autocrine proteins binding to LDL receptor-related proteins (LRPs), Frizzled (Fzd) receptors and/or receptor tyrosine kinase-like orphan receptors (RORs) $(3,4)$. Wnt5a, a non-canonical Wnt ligand, binds to Fzd2 and subsequently inhibits Wnt3a-induced LRP6 activation and $\beta$-catenin signaling (5). The Fzd5 receptor can transduce Wnt5a signaling to $\beta$-catenin and induce the formation of a secondary axis in Xenopus embryos $(6,7)$.

Previous studies have demonstrated that Wnt5a activates dishevelled 2/disheveled-associated activator of morphogenesis 1 (DAAM1)/RhoA, and subsequently enhances cell migration in breast cancer and cell invasion in glioblastoma $(8,9)$. Wnt5a and type IV collagen were revealed to activate DAAM1 and promote cell migration and haptotaxis in breast cancer, respectively $(8,10)$. Wnt5a enhanced leukemia cell chemotaxis and proliferation by inducing the oligomerization of ROR1 and ROR2 and activating RhoA/Rac1 (11). Although it has been reported that Wnt5a/ROR2 phosphorylates PI3K/Akt, activates RhoA and promotes cell migration in osteosarcoma $(12,13)$, the role of ROR1 in the Wnt5a-induced migration of osteosarcoma cells is still unknown.

The present study aimed to reveal whether ROR1 activates DAAM1 and promotes the migration of osteosarcoma cells in response to Wnt5a. It was demonstrated that ROR1/ROR2dependent DAAM1 and parallel PI3K $\alpha /$ Akt signaling activated RhoA and regulated Wnt5a-induced osteosarcoma cell migration. These data indicated ROR1/ROR2 may be novel clinical targets in inhibiting osteosarcoma migration and decreasing mortality.

\section{Materials and methods}

Cell lines. MG-63 and U2OS human osteosarcoma cells were purchased from Cell Bank (Shanghai, China) and cultured in DMEM supplemented with $10 \%$ bovine serum 
(HyClone; GE Healthcare Life Sciences), $0.5 \mu \mathrm{g} / \mathrm{ml}$ penicillin and streptomycin. All cell lines were verified monthly to be mycoplasma-negative.

Short hairpin (sh)RNAs targeted to ROR2 (5'-AACTC TGAAAGGTTACTTTCTGA-3') and DAAM1 (5'-GCCACT TTGTATCCTATCAGG-3'), GFP-RhoA-V14 and wild-type (WT) ROR2 constructs were transfected into osteosarcoma cells using Lipofectamine ${ }^{\circledR} 3000$ (Invitrogen; Thermo Fisher Scientific, Inc.).

Western blotting. MG-63 and U2OS cells, incubated in medium containing $100 \mathrm{ng} / \mathrm{ml} \mathrm{Wnt5a}$, were treated with $1 \mu \mathrm{mol} / \mathrm{l}$ Rho inhibitor CCG-1423 (Selleck), $1 \mathrm{nmol} / 1 \mathrm{PI} 3 \mathrm{~K} \alpha$ inhibitor HS-173 (APExBIO), $1 \mu \mathrm{g} / \mathrm{ml}$ anti-ROR $1 \mathrm{mAb}$ or vehicle for $1 \mathrm{~h}$. Then, the total protein of osteosarcoma cells was extracted using ice-cold RIPA lysis buffer (14). Cellular lysates were centrifuged at 12,000 $\mathrm{x}$ g for $30 \mathrm{~min}$ in a cooler. The protein concentration was determined by BCA method. Proteins were separated in $10 \%$ gels and then transferred to polyvinylidene difluoride membranes. The membranes were blocked in 5\% BSA for $30 \mathrm{~min}$ at room temperature. The primary antibodies employed were as follows: Rabbit anti-Akt antibody (60 kDa; dilution 1:1,000; product \#4691) and rabbit anti-phospho-Akt (p-Ser473) antibody (60 kDa; dilution 1:1,000; product \#4058, both from Cell Signaling Technology, Inc.; Fig. 4A), mouse anti-phospho-Akt (60 kDa; dilution 1:1,000; p-Ser473) antibody (cat. no. 66444 ; Fig. $4 \mathrm{D}$ ), anti- $\beta$-actin ( $45 \mathrm{kDa}$; dilution 1:5,000; cat. no. 60008) and anti-ROR1 (130 kDa; dilution 1:1,000; cat. no. 20629; all from ProteinTech Group, Inc.), anti-ROR2 (135 kDa; dilution 1:1,000; product no. 88639), anti-flag (product no. 14793, dilution 1:1,000), anti-GST (product no. 2624; dilution 1:1,000; all from Cell Signaling Technology, Inc.) and anti-DAAM1 (122 kDa; dilution 1:1,000; cat. no. sc-100942; Santa Cruz Biotechnology, Inc.) antibodies. Quantitative analysis of each blot was performed with densitometric scanning. The bands were visualized using enhanced chemiluminescent (ECL) kit (Tanon, China) and densitometry analysis was performed using Tanon software (Shanghai, China).

Pulldown assays and immunoprecipitation (IP). The measurement of DAAM1 activation was performed as previously described (10). The bacterially-expressed flagROR2 and GST-ROR1 were purified using Pierce Anti-flag Magnetic Agarose Beads (Thermo Fisher Scientific, Inc.) and GST-Sepharose 4B (GE Healthcare), respectively. Beads that had captured the targeted proteins were subjected to western blot analysis, which was performed according to the western blotting protocol aforementioned.

Equal volumes of total cellular protein extractions were subjected to Rho GTPase activation assays (Cytoskeleton, Inc.). The detailed procedure for the G-LISA assays was previously described (10).

The lysates of MG-63 cells were centrifuged at $12,000 \mathrm{x} \mathrm{g}$ for $15 \mathrm{~min}$. The supernatants of cellular lysates were incubated with $\operatorname{lgG}$ or ROR2 antibodies for $12 \mathrm{~h}$. Protein A/G-conjugated agarose beads (Pierce; Thermo Fisher Scientific, Inc.) were mixed with the cell lysates and ROR2 antibodies and maintained under agitation for $2 \mathrm{~h}$. After washing with lysis buffer four times, the beads were subjected to western blotting.
Boyden chamber assays. A Boyden chamber system $(8.0-\mu \mathrm{m}$; Costar; Corning, Inc.) was used to perform the cell migration assays. A total of $1 \times 10^{5}$ cells with $5 \mu \mathrm{g} / \mathrm{ml} \mathrm{BSA}$ was seeded into the Boyden chambers and allowed to migrate for $5 \mathrm{~h}$. Subsequently, the migratory cells in the lower compartments of the Boyden chambers were stained with $0.2 \%$ crystal violet for $20 \mathrm{~min}$. The number of migratory cells was counted under a brightfield microscope (Mshot MF53; Micro-shot Technology Co., Ltd.).

Actin cytoskeleton staining. Cells grown on glass slides were fixed in $4 \%$ paraformaldehyde for $15 \mathrm{~min}$. Then, the cells were permeabilized by $0.2 \%$ Triton X-100 and blocked in $1 \%$ BSA for $1 \mathrm{~h}$. Next, TRITC-labeled phalloidin $(5 \mu \mathrm{g} / \mathrm{ml}$; Sigma-Aldrich; Merck $\mathrm{KGaA}$ ) was used to stain the actin cytoskeleton for $1 \mathrm{~h}$. The nucleus was stained with DAPI. Fluorescent images were captured using a Zeiss confocal microscope (LSM710; Zeiss AG).

Statistical analysis. One-way ANOVA or a Student's t-test were used to perform statistical analyses, with SPSS 22.0 software (IBM Corp.). Bar and scatter charts present the mean \pm SD of five independent replicates. $\mathrm{P}<0.05$ was considered to indicate a statistically significant difference.

\section{Results}

ROR1 directly binds to ROR 2 and mediates Wnt5a-induced cell migration. Wnt5a can induce ROR1 and ROR2 heterooligomerization in leukemia cells (11). The present study verified whether ROR1 was able to physically bind to ROR2 in osteosarcoma cells. The IP assay demonstrated that ROR2 bound to ROR1 in MG-63 osteosarcoma cells (Fig. 1A). Moreover, it was revealed that purified GST-tagged ROR1 was directly bound to flag-tagged ROR2 (Fig. 1B).

Since Wnt5a/ROR2 has been revealed to promote cell migration in osteosarcoma (13), it was hypothesized that ROR1 may play a similar role to that of ROR2 in osteosarcoma cell migration. As anticipated, the inhibition of ROR1 signaling by ROR $1 \mathrm{mAb}$ treatment abolished the migration of Wnt5a-treated osteosarcoma cells (Fig. 1C). Overexpression of shRNA-resistant WT ROR2 rescued the ROR2-shRNAmediated, but not the ROR1 mAb-induced, decrease in cell migration (Fig. 1D). The treatment with the ROR $1 \mathrm{mAb}$, the transfection of ROR2-shRNA, and ROR2-shRNA plus ROR1 $\mathrm{mAb}$ resulted in an equal significant decrease in cell migration (Fig. 1D). These results demonstrated that ROR1 and ROR2 receptors mediated the Wnt5a-induced migration of osteosarcoma cells.

ROR1/ROR2 activates RhoA and mediates cell migration. The finding that RhoA mediates Wnt5a-induced cell migration in osteosarcoma (12) prompted the study of whether Wnt5a/ROR1/ROR2 signaling activated RhoA in osteosarcoma. G-LISA assays revealed a significant decrease in the activation of RhoA, but not that of Rac1 or Rac2, in osteosarcoma cells treated with ROR $1 \mathrm{mAb}$ and knockdown of ROR 2 by ROR2-shRNA transfection (Fig. 2A and B). Overexpression of shRNA-resistant WT ROR2 rescued ROR2-shRNA-mediated, but not ROR1 mAb-induced, decrease of RhoA activation 
A

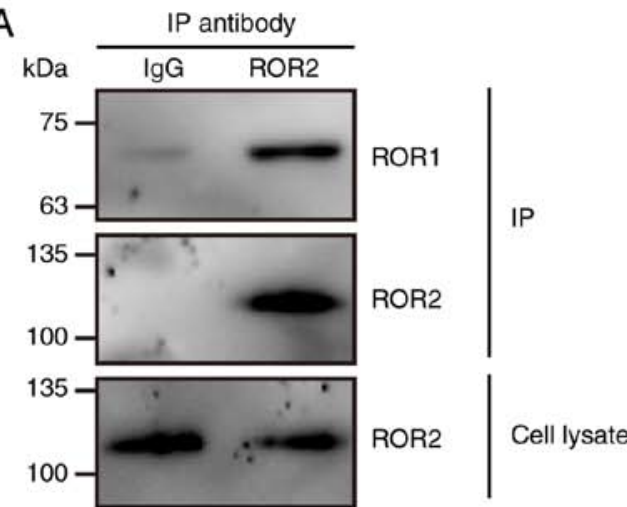

B

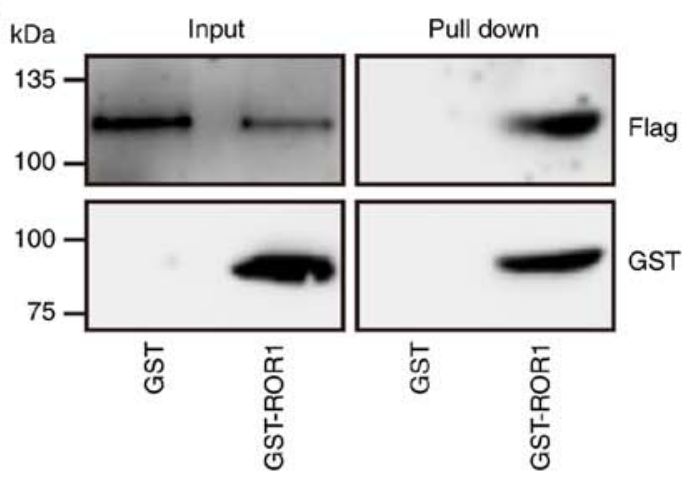

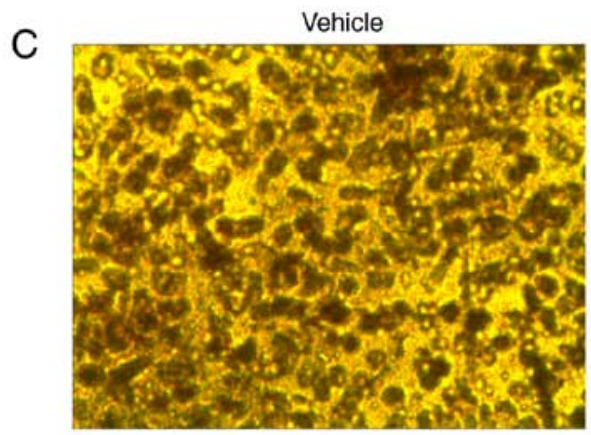
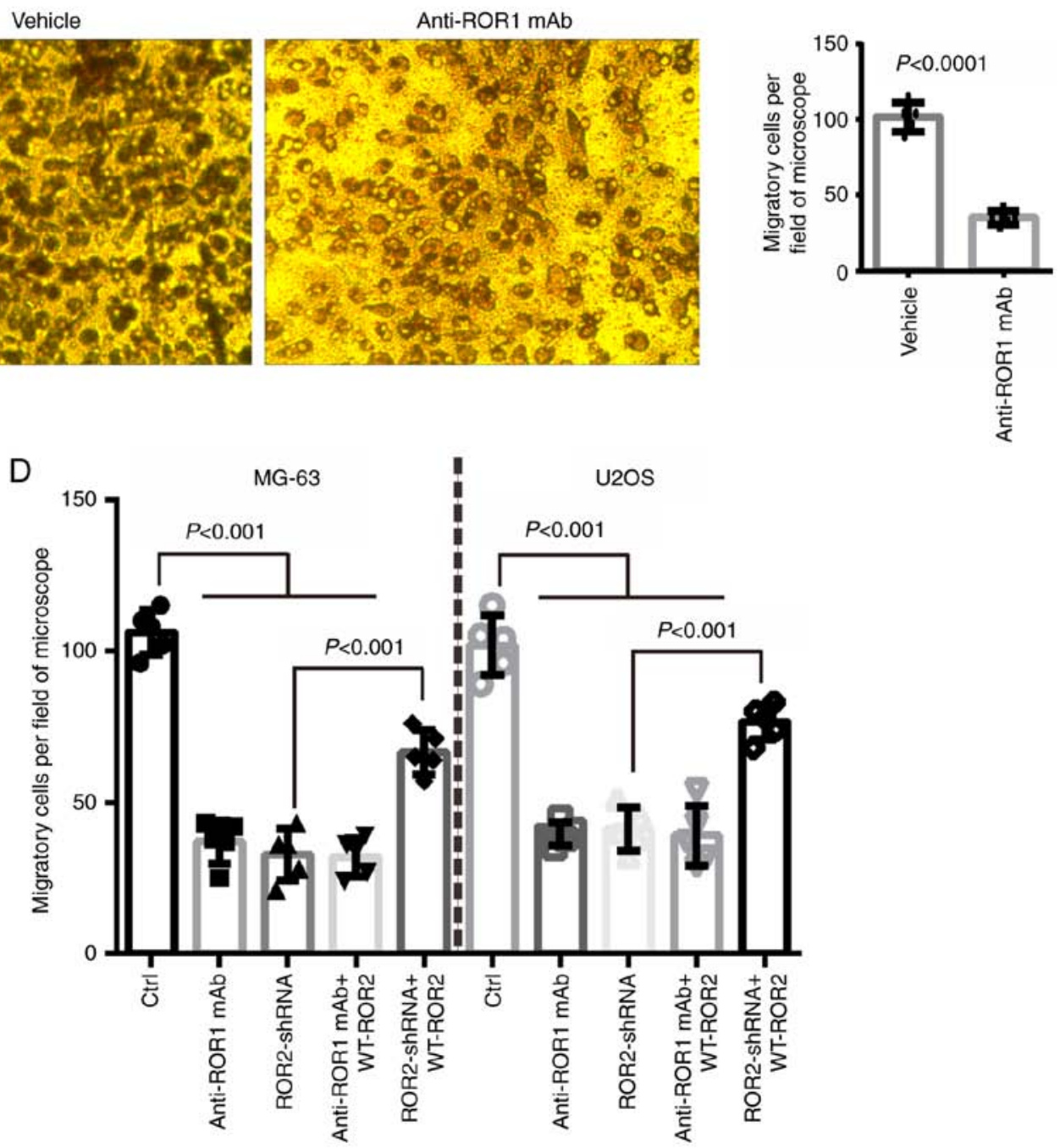

Figure 1. ROR1 directly binds to ROR2 and mediates Wnt5a-induced osteosarcoma cell migration. (A) The lysate of MG-63 osteosarcoma cells was subjected to immunoprecipitation with an antibody against ROR2, followed by immunoblotting with ROR1 or ROR2 antibodies. (B) ROR1 directly bound to ROR2 . Purified GST or GST-ROR1 was incubated with purified Flag-tagged full-length ROR2. The amounts of Flag-ROR2 co-purified with GST or GST-ROR1 (pulldown) were analyzed by immunoblotting for anti-Flag or anti-GST. (C) Cell migration was significantly abolished by ROR1 mAb. MG-63 cells were incubated with ROR1 mAb and then subjected to cell migration assays. The cells incubated in the medium containing $100 \mathrm{ng} / \mathrm{ml} \mathrm{Wnt} 5 \mathrm{a}$ were allowed to migrate for $5 \mathrm{~h}$. Magnification, x20. (D) MG-63 and U2OS osteosarcoma cells were treated with ROR1 mAb, transfected with ROR2 shRNA, treated with combination ROR1-mAb treatment with WT-ROR2 transfection, or treated with combination ROR2 shRNA with shRNA-resistant WT-ROR2 transfection, and then subjected to cell migration assays. The cells incubated in medium containing $100 \mathrm{ng} / \mathrm{ml} \mathrm{Wnt5a}$ were allowed to migrate for $5 \mathrm{~h}$. The number of migratory cells/field of microscope was counted. ROR1, receptor tyrosine kinase-like orphan receptor 1; ROR2, receptor tyrosine kinase-like orphan receptor 2; mAb, monoclonal antibody; shRNA, short hairpin RNA; WT, wild-type.

(Fig. 2A). ROR1 mAb, ROR2-shRNA, and ROR2-shRNA plus ROR1 $m A b$ resulted in an equal significant reduction of RhoA activity (Fig. 2A).
Constitutively activated RhoA (RhoA-CA) was used to verify the accelerated effect of RhoA activity on cellular motility. Overexpression of RhoA-CA completely rescued 
A

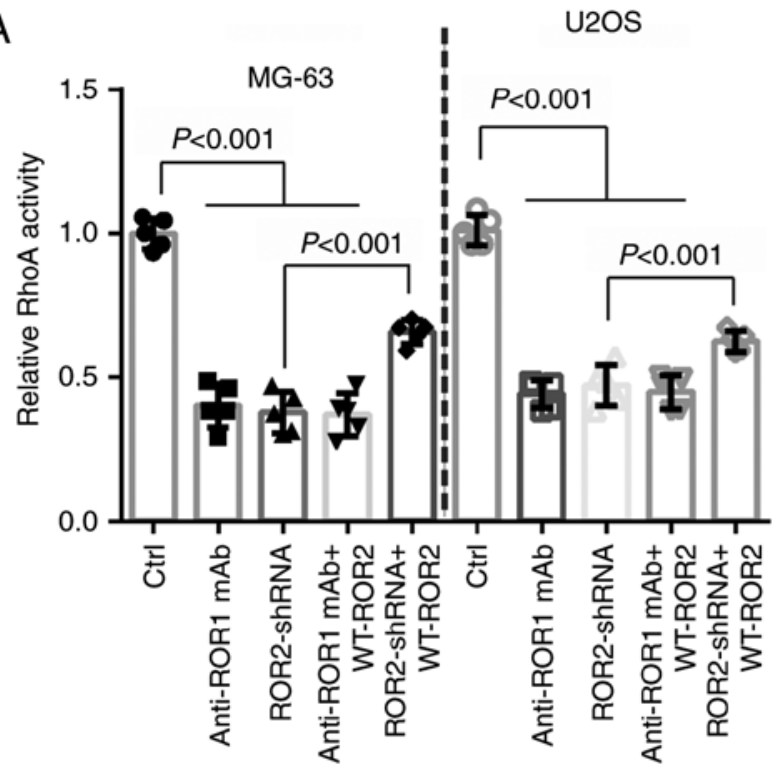

B

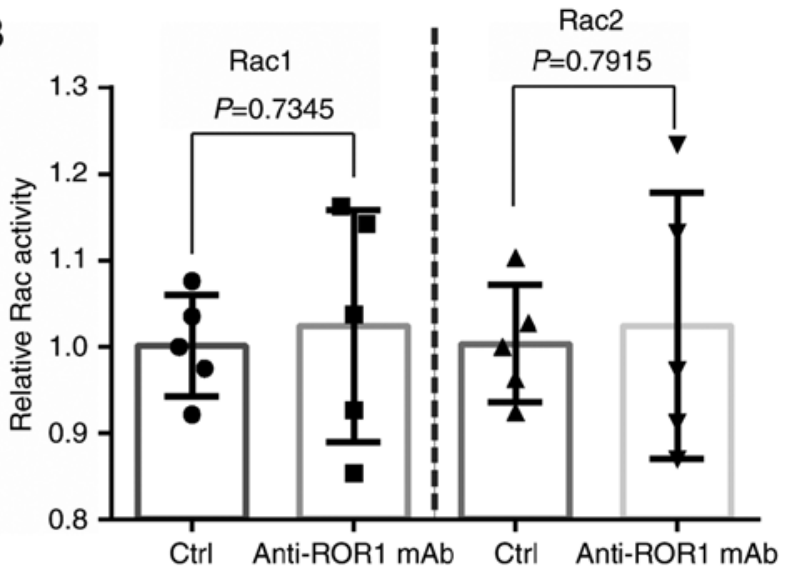

C

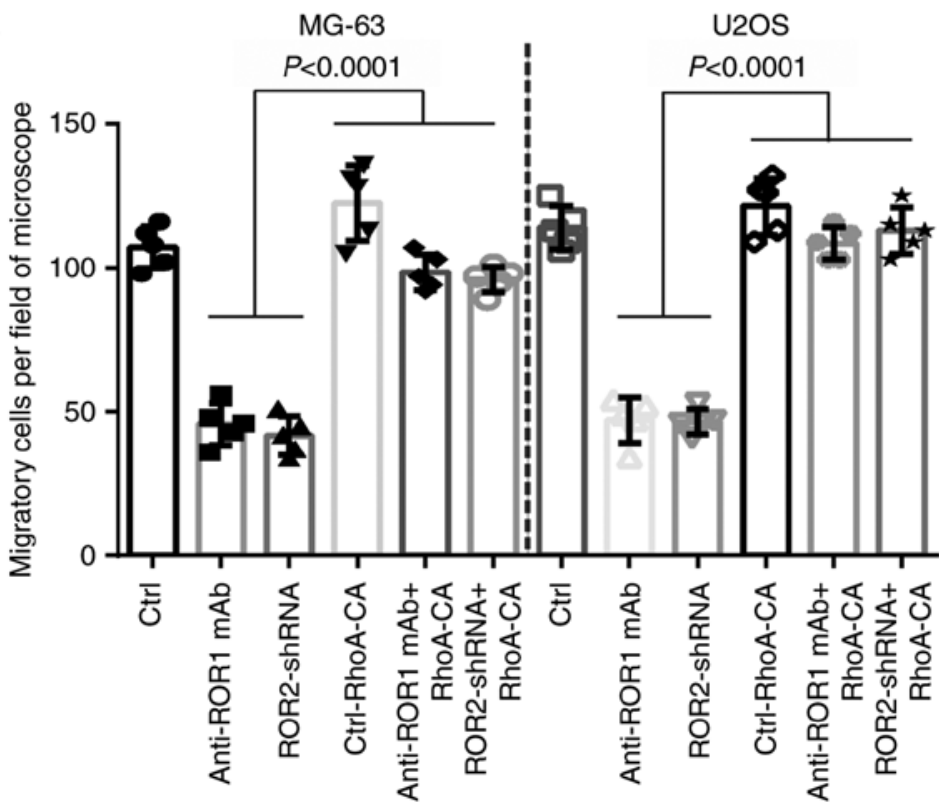

Figure 2. Wnt5a-induced RhoA activation is regulated by ROR1/ROR2, and required for osteosarcoma cell migration. (A) MG-63 and U2OS osteosarcoma cells were treated with ROR1 mAb, transfected with ROR2 shRNA, treated with combination ROR1-mAb treatment with WT-ROR2 transfection, or treated with combination ROR2-shRNA with shRNA-resistant WT-ROR2 transfection, and then the RhoA activation was assessed using G-LISA assays. The cells were incubated in medium containing $100 \mathrm{ng} / \mathrm{ml}$ Wnt5a. (B) MG-63 cells were treated with ROR1 mAb, and then the activation of Rac1 and Rac2 was assessed using G-LISA assays. The cells were incubated in medium containing $100 \mathrm{ng} / \mathrm{ml} \mathrm{Wnt5a.} \mathrm{(C)} \mathrm{MG-63} \mathrm{and} \mathrm{U2OS} \mathrm{osteosarcoma} \mathrm{cells} \mathrm{were} \mathrm{treated} \mathrm{with} \mathrm{ROR1}$ mAb, transfected with ROR2 shRNA, transfected with constitutively RhoA-CA, treated with combination ROR1-mAb treatment with RhoA-CA transfection, or treated with combination ROR2-shRNA with RhoA-CA transfection, and then subjected to cell migration assays. The cells incubated in medium containing $100 \mathrm{ng} / \mathrm{ml}$ Wnt5a were allowed to migrate for $5 \mathrm{~h}$. The number of migratory cells/field of microscope was counted. ROR1, receptor tyrosine kinase-like orphan receptor 1; ROR2, receptor tyrosine kinase-like orphan receptor 2; mAb, monoclonal antibody; shRNA, short hairpin RNA; WT, wild-type; RhoA-CA, activated RhoA.

the ROR1 mAb- or ROR2-shRNA-mediated decrease of cell migration in osteosarcoma cells (Fig. 2C). These results demonstrated that RhoA was activated by Wnt5a/ROR1/ROR2 and mediated osteosarcoma cell migration.

DAAM1 mediates the migration of osteosarcoma cells. The activation of RhoA was assessed in DAAM1-silenced osteosarcoma cells. An $\sim 0.5$-fold decrease was observed in DAAM1-silenced osteosarcoma cells (Fig. 3A). CCG-1423 blocked the RhoA activity, but failed to alter DAAM1 activity
(Fig. 3B). These data indicated that RhoA acted as the downstream target of DAAM1.

The present study verified whether the activation of DAAM1 was dependent on ROR1 and ROR2 receptors in osteosarcoma cells. The activation of DAAM1 was decreased after ROR1 mAb treatment or ROR2 downregulation (Fig. 3C). The migration of DAAM1-silenced osteosarcoma cells was significantly delayed, which was reversed by RhoA-CA overexpression (Fig. 3D). These results indicated that ROR1 and ROR2 may activate DAAM1/RhoA and promote cell migration in osteosarcoma. 
A

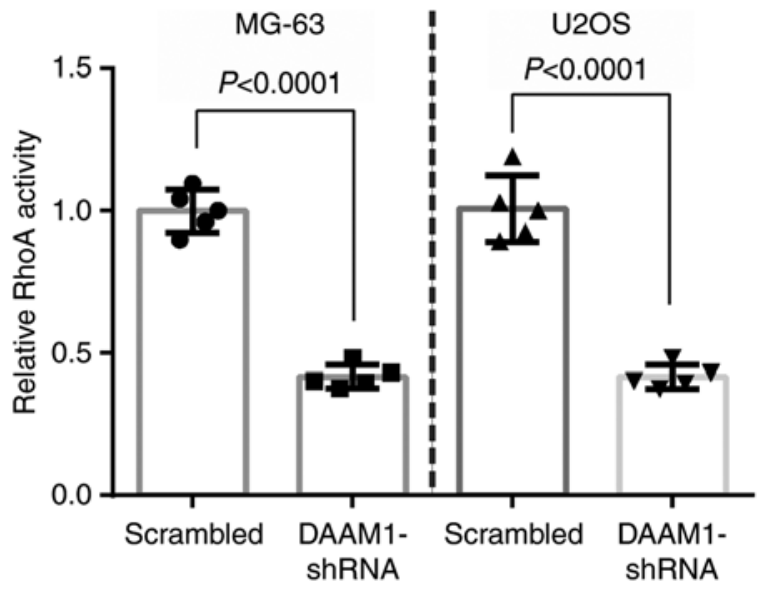

C

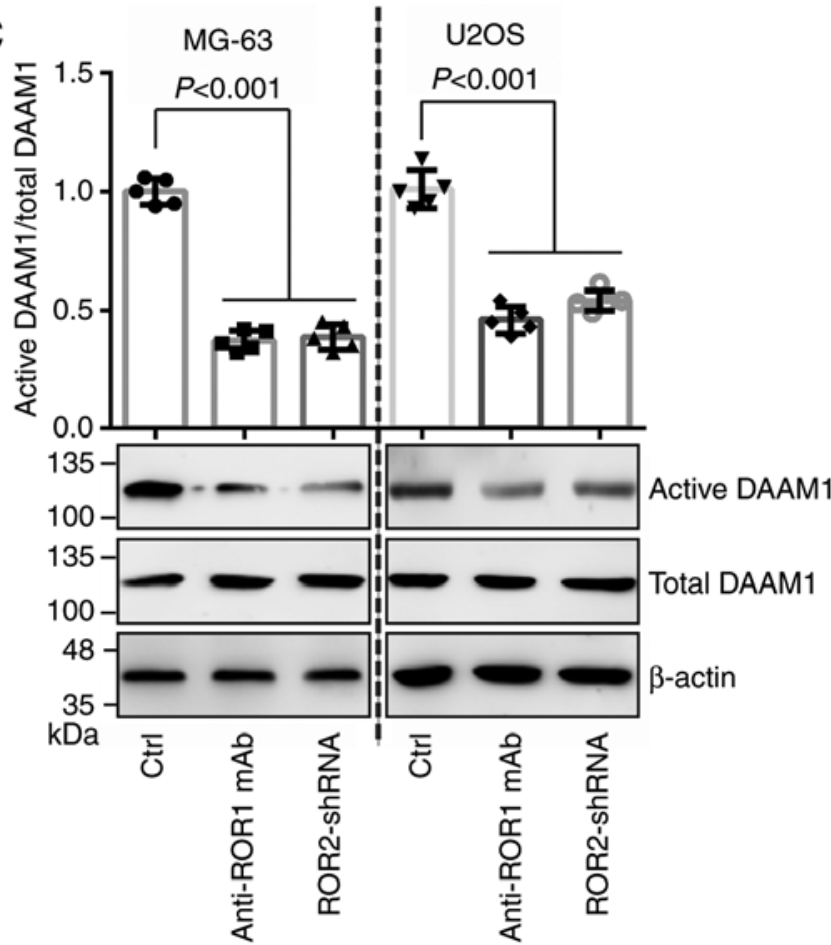

B

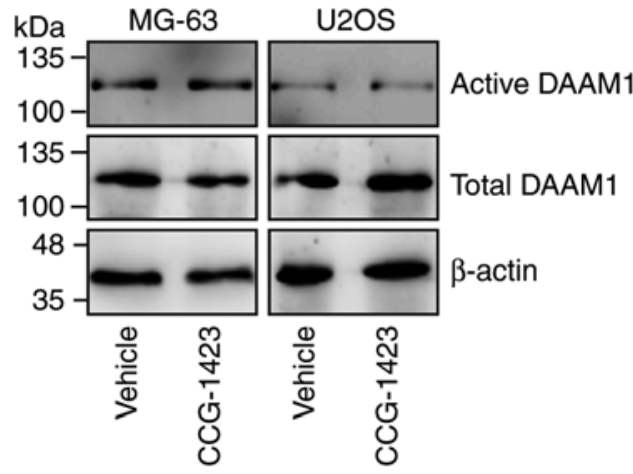

D

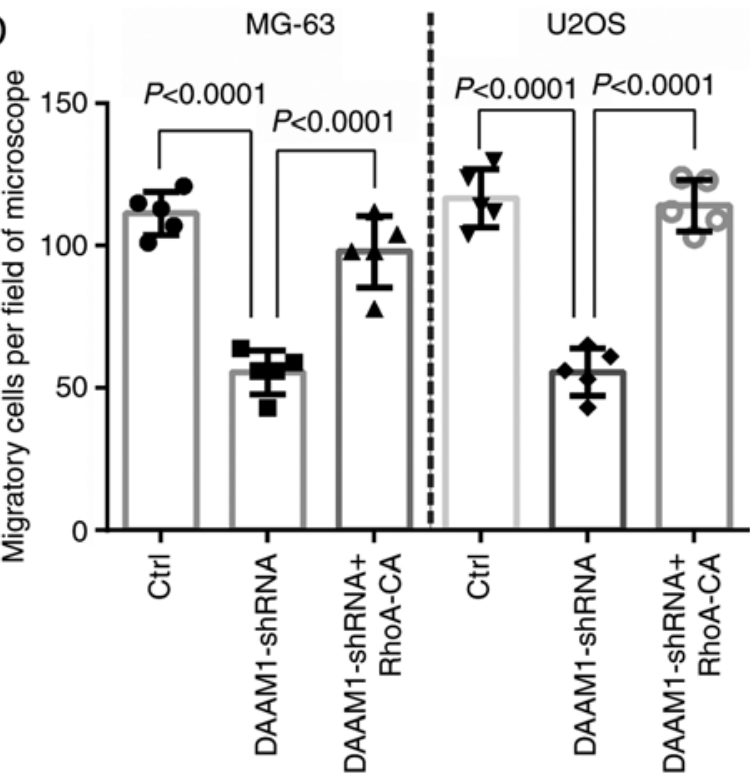

Figure 3. DAAM1 acts upstream of RhoA and mediates Wnt5a-induced osteosarcoma cell migration. (A) MG-63 and U2OS osteosarcoma cells were transfected with DAAM1-shRNA or scrambled shRNA, and then the RhoA activity was examined using G-LISA assays. The cells were incubated in medium containing $100 \mathrm{ng} / \mathrm{ml}$ Wnt5a. (B) DAAM1 activation was not altered by the treatment with the RhoA-specific inhibitor CCG-1423. MG-63 and U2OS cells incubated in medium containing $100 \mathrm{ng} / \mathrm{ml}$ Wnt5a were treated with $1 \mu \mathrm{mol} / 1 \mathrm{CCG}-1423$ or vehicle. Cellular lysates were assayed for the active DAAM1 via a pulldown assay using a GST-RhoA as a bait. (C) DAAM1 acted as the downstream target of ROR1 and ROR2 receptors. MG-63 and U2OS cells incubated in medium containing $100 \mathrm{ng} / \mathrm{ml}$ Wnt5a were treated with ROR1 mAb or transfected with ROR2-shRNA. Cellular lysates were assayed for the active DAAM1 via a pulldown assay using a GST-RhoA as a bait. (D) MG-63 and U2OS cells were transfected with DAAM1-shRNA or combination DAAM1-shRNA with RhoA-CA transfection, and then subjected to migration assays. The cells incubated in medium containing $100 \mathrm{ng} / \mathrm{ml}$ Wnt5a were allowed to migrate for $5 \mathrm{~h}$. The number of migratory cells/field of microscope was counted. DAAM1, disheveled-associated activator of morphogenesis 1; ROR1, receptor tyrosine kinase-like orphan receptor 1; ROR2, receptor tyrosine kinase-like orphan receptor 2; shRNA, short hairpin RNA; mAb, monoclonal antibody.

DAAM1 and PI3Ka/Akt are parallel signaling pathways mediating Wnt5a-induced cell migration. The pulldown assay and immunoblotting demonstrated that knockdown of DAAM1 did not alter PI3Ka/Akt activity (Fig. 4A). Conversely, the inhibition of PI3K $\alpha /$ Akt activity by treatment with HS-173 also did not change DAAM1 activity (Fig. 4B). The combination of HS-173 treatment with DAAM1-shRNA transfection further delayed Wnt5a-induced cell migration compared with HS-173 treatment or DAAM1-shRNA transfection alone (Fig. 4C). Anti-ROR1 mAb markedly blocked the phosphorylation of Akt in osteosarcoma cells (Fig. 4D). Thus, these results indicated that DAAM1 and PI3Ka/Akt are parallel signaling pathways mediating Wnt5a-induced cell migration.

RORI/ROR 2 regulates the microfilament assembly in osteosarcoma cells. Lastly, the present study validated whether ROR1/2 signaling was involved in the assembly of microfilaments in osteosarcoma cells. Fluorescent phalloidin was used to stain filamentous actin, thereby displaying its distribution in MG-63 cells. ROR1 mAb eliminated the assembly of 

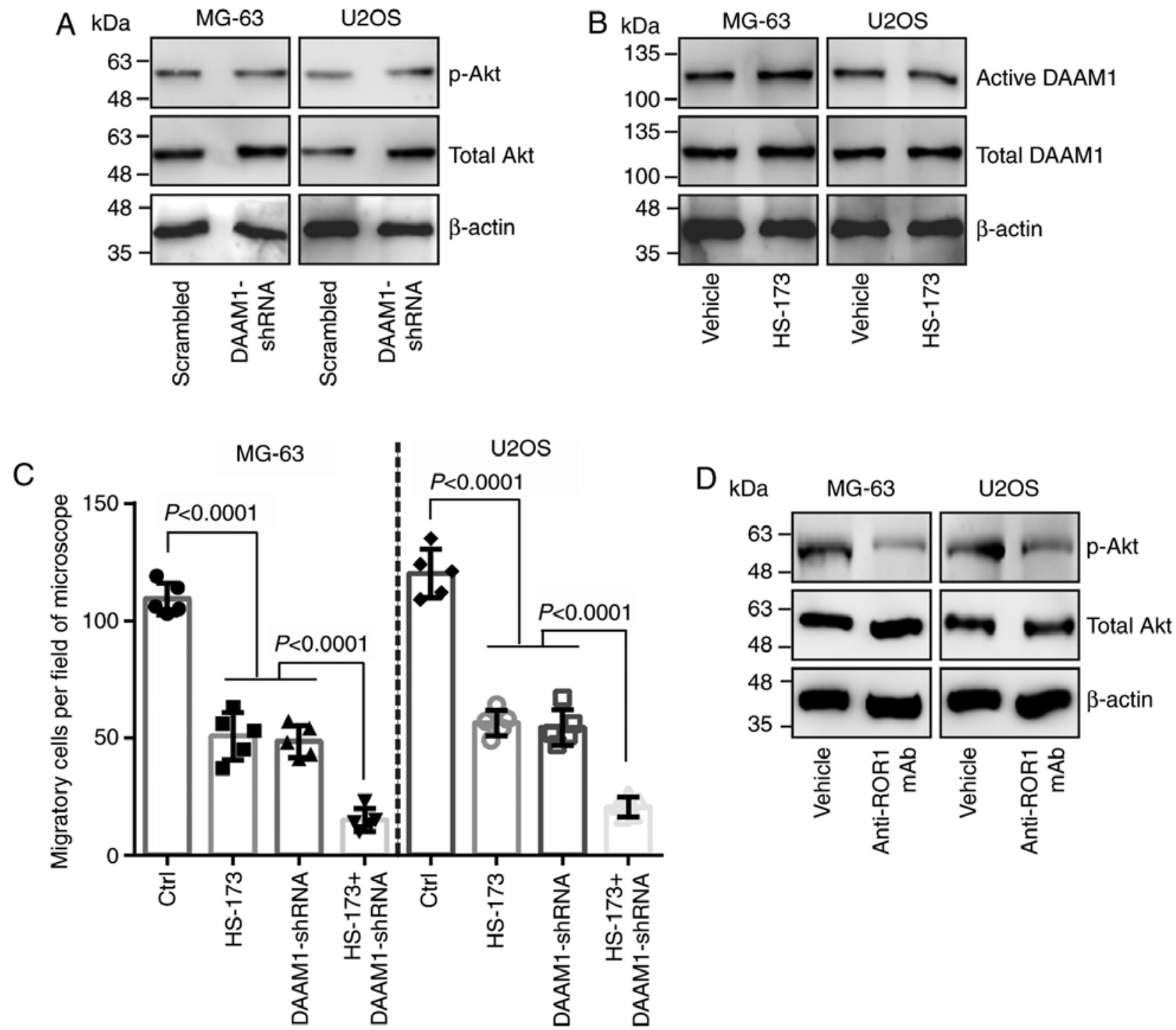

Figure 4. DAAM1 and PI3Ka/Akt are parallel signaling pathways mediating Wnt5a-induced cell migration. (A) MG-63 and U2OS osteosarcoma cells were transfected with DAAM1-shRNA or scrambled shRNA and then subjected to western blotting to assess the phosphorylation of Akt (p-Ser473). The cells were incubated in medium containing $100 \mathrm{ng} / \mathrm{ml} \mathrm{Wnt5a}$. (B) MG-63 and U2OS cells were incubated in medium containing $100 \mathrm{ng} / \mathrm{ml} \mathrm{Wnt5a}$ were treated with $1 \mathrm{nmol} / \mathrm{l} \mathrm{HS}-173$ (PI3K $\alpha$ inhibitor) or vehicle. Cellular lysates were assayed for active DAAM1 via a pulldown assay using GST-RhoA as a bait. (C) MG-63 and U2OS cells were treated with $1 \mathrm{nmol} / 1$ HS-173, transfected with DAAM1-shRNA, or treated with combination HS-173 treatment with DAAM1-shRNA transfection, and then subjected to cell migration assays. The cells incubated in medium containing $100 \mathrm{ng} / \mathrm{ml} \mathrm{Wnt5a}$ were allowed to migrate for $5 \mathrm{~h}$. The number of migratory cells/field of microscope was counted. (D) MG-63 and U2OS cells were treated with anti-ROR1 mAb or vehicle, and then subjected to western blotting to measure the phosphorylation of Akt (p-Ser473). The cells were incubated in medium containing $100 \mathrm{ng} / \mathrm{ml}$ Wnt5a. DAAM1, disheveledassociated activator of morphogenesis 1; ROR1, receptor tyrosine kinase-like orphan receptor 1; ROR2, receptor tyrosine kinase-like orphan receptor 2; shRNA, short hairpin RNA; mAb, monoclonal antibody.

microfilaments and reduced microfilament length (Fig. 5). Moreover, ROR2-shRNA disrupted the formation of microfilaments and shortened microfilament length (Fig. 5). These results indicated that ROR1/ROR2 may modulate the assembly of microfilaments of osteosarcoma cells.

\section{Discussion}

ROR1 and ROR2, two confirmed receptors for the Wnt5a ligand, mediate non-canonical Wnt signaling regulating embryo/organ development and tumor metastasis (15). UC-961 (cirmtuzumab) is a humanized IgG1 monoclonal antibody that directly binds to ROR1 and completely blocks ROR1 signaling (16-19), and has been demonstrated to be effective and safe in a phase I trial for patients with progressive, relapsed or refractory chronic lymphocytic leukemia (20). The present study revealed that ROR1 mAb markedly blocked the activation of DAAM1 and RhoA, the formation of microfilaments and the migration of osteosarcoma cells. ROR1 may be a novel therapeutic target to delay osteosarcoma metastasis.

Wnt5a has been revealed to facilitate the dimerization of ROR1/ROR2, and thus enhance leukemia metastasis and proliferation $(11,15)$. ROR2 was revealed to mediate Wnt5a-induced cell migration through PI3K/Akt phosphorylation and RhoA activation in osteosarcoma $(12,13,21)$. In the present study, it was revealed that the silencing of ROR2 significantly suppressed the activation of DAAM1 and RhoA, the formation of microfilaments, and cell migration in 
A
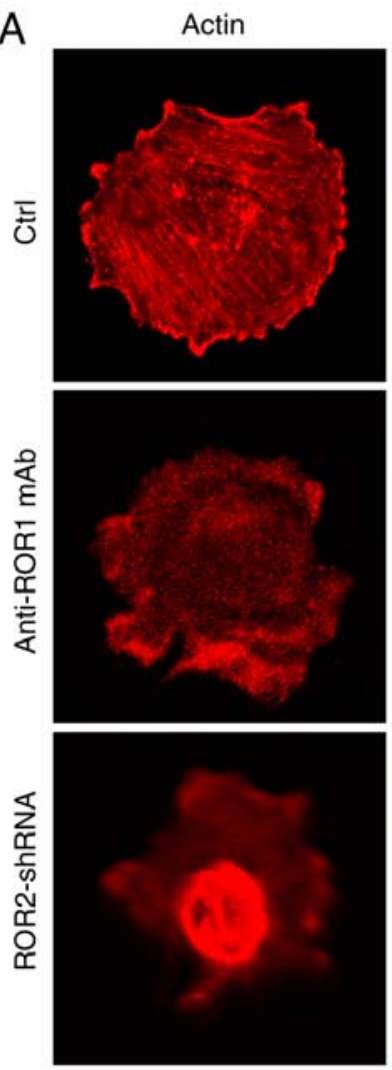

DAPI
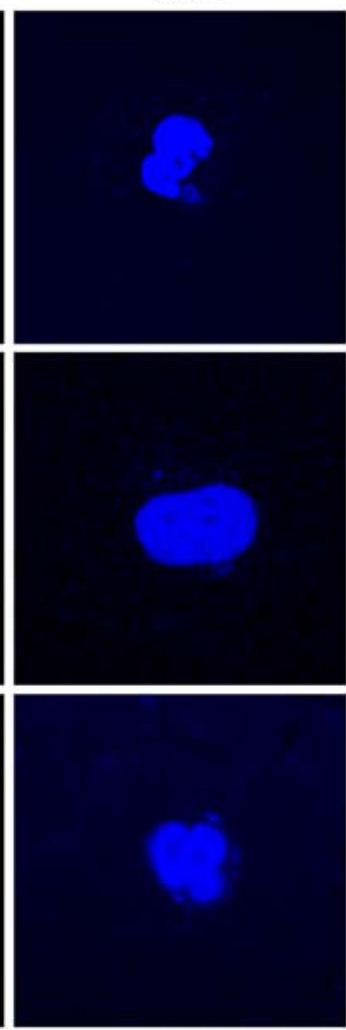
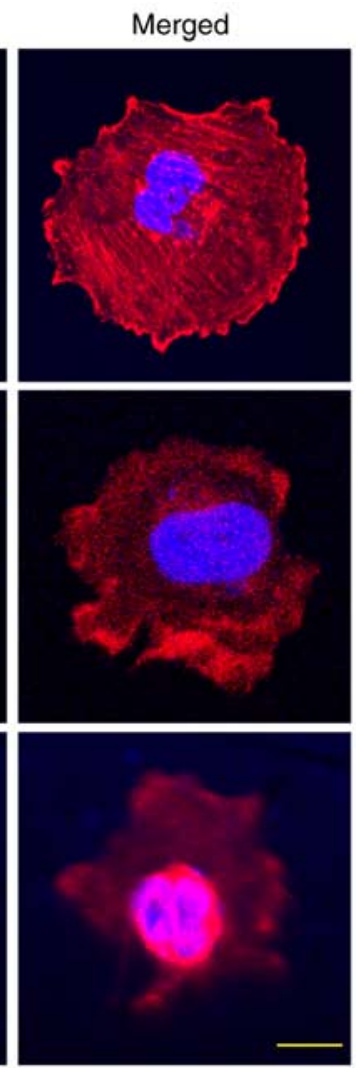

B
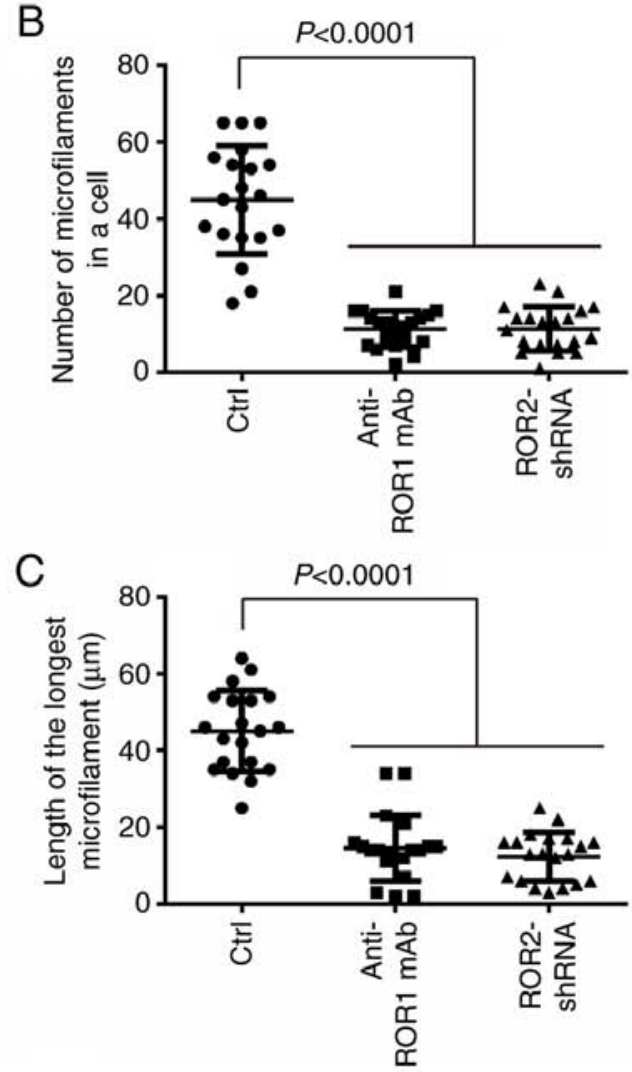

Figure 5. ROR1 and ROR2 participate in the Wnt5a-induced rearrangement of microfilaments. (A) Blocking ROR1/ROR2 signaling by ROR1 mAb treatment or ROR2-shRNA transfection disrupted the formation of microfilaments. MG-63 osteosarcoma cells treated with ROR1 mAb or transfected with ROR2shRNA were grown on coverslips and incubated in medium containing $100 \mathrm{ng} / \mathrm{ml}$ Wnt5a. Subsequently, the cells were fixed and stained with phalloidin and DAPI to reveal the filamentous actin organization and nucleus location. Scale bar, $10 \mu \mathrm{m}$. Magnification, $\mathrm{x} 40$. (B and C) The number of microfilaments in a cell and the length of the longest microfilament were determined in MG-63 cells. ROR1, receptor tyrosine kinase-like orphan receptor 1; ROR2, receptor tyrosine kinase-like orphan receptor 2; shRNA, short hairpin RNA; mAb, monoclonal antibody.

osteosarcoma. The equivalent effect of ROR1 mAb, ROR2shRNA and ROR1 mAb plus ROR2-shRNA on cell motility indicated that ROR1/ROR2 dimers may mediate tumor cell migration in response to Wnt5a. Further studies are required to consolidate the potential role of ROR1/ROR2 in vivo. The PI3K/Akt signaling pathway involves a group of phosphatases, which regulate diverse cellular functions including metabolism, growth, proliferation, survival, transcription and protein synthesis $(22,23)$. Wnt5a has been revealed to activate RhoA and subsequently promotes the invasion/migration of osteosarcoma, gastric cancer, breast cancer and glioblastoma cells $(8,9,12,13,24,25)$. PI3K/Akt activated RhoA activity and subsequently enhanced Wnt5a-induced cell migration in both gastric cancer and osteosarcoma cells $(12,25)$. The present results demonstrated that anti-ROR $1 \mathrm{mAb}$ blocked the signaling of PI3K $\alpha /$ Akt of osteosarcoma cells. A limitation of the present study is that the knockdown of ROR1 on the PI3K $\alpha /$ Akt pathway was not performed. The knockdown of DAAM1 downregulated RhoA activity and cell migration in osteosarcoma. The downregulation of RhoA activity, microfilament formation and cell migration in DAAM1silenced cells indicated that DAAM1 played a relevant in Wnt5a/ROR1/ROR2 by activating RhoA. Moreover, it was demonstrated that DAAM1 and PI3K $\alpha / A k t$ are parallel signaling pathways transducing ROR1/2 signaling and mediating Wnt5a-induced cell migration.
In summary, the present study clarified the association of Wnt5a/ROR1 signaling and osteosarcoma migration. Wnt5a/ROR1/2 can activate RhoA via DAAM1 and the parallel signaling pathway PI3Ka/Akt, and regulate the migration of osteosarcoma cells.

\section{Acknowledgements}

Not applicable.

\section{Funding}

The present study was supported by grants from Jiangsu Province's 333 Research Project to BD (BRA2016208), the Science and Technology Bureau and Commission of Health and Family Planning of Yancheng City to BD (YK2017062), the Science and Technology Foundation of Nanjing Medical University (2017NJMU001) to TY, and the Project funded by China Postdoctoral Science Foundation (2018M630602) and the Science and Technology Bureau of Changzhou (CJ20190061) to AZ.

\section{Availability of data and materials}

The datasets used during the present study are available from the corresponding author upon reasonable request. 


\section{Authors' contributions}

AZ designed the project and wrote the paper. BD and YS performed the experiments. TY analyzed the data. All authors read and approved the manuscript and agree to be accountable for all aspects of the research in ensuring that the accuracy or integrity of any part of the work are appropriately investigated and resolved.

\section{Ethics approval and consent to participate}

Not applicable.

\section{Patient consent for publication}

Not applicable.

\section{Competing interests}

The authors declare that they have no competing interests.

\section{References}

1. Mirabello L, Troisi RJ and Savage SA: International osteosarcoma incidence patterns in children and adolescents, middle ages and elderly persons. Int J Cancer 125: 229-234, 2009.

2. Misaghi A, Goldin A, Awad M and Kulidjian AA: Osteosarcoma: A comprehensive review. SICOT J 4: 12, 2018.

3. Nusse R, van Ooyen A, Cox D, Fung YK and Varmus H: Mode of proviral activation of a putative mammary oncogene (int-1) on mouse chromosome 15. Nature 307: 131-136, 1984.

4. Nusse R: Wnt signaling in disease and in development. Cell Res 15: 28-32, 2005.

5. Sato A, Yamamoto H, Sakane H, Koyama H and Kikuchi A: Wnt5a regulates distinct signalling pathways by binding to Frizzled2. EMBO J 29: 41-54, 2010.

6. He X, Saint-Jeannet JP, Wang Y, Nathans J, Dawid I and Varmus H: A member of the Frizzled protein family mediating axis induction by Wnt-5A. Science 275: 1652-1654, 1997.

7. Ishikawa T, Tamai Y, Zorn AM, Yoshida H, Seldin MF, Nishikawa S and Taketo MM: Mouse Wnt receptor gene Fzd5 is essential for yolk sac and placental angiogenesis. Development 128: 25-33, 2001.

8. Zhu Y,Tian Y,Du J,Hu Z, Yang L, Liu J and Gu L: Dvl2-dependent activation of Daam1 and RhoA regulates Wnt5a-induced breast cancer cell migration. PLoS One 7: e37823, 2012.

9. Liu G, Yan T, Li X, Sun J, Zhang B, Wang H and Zhu Y: Daam1 activates RhoA to regulate Wnt5a-induced glioblastoma cell invasion. Oncol Rep 39: 465-472, 2018.

10. Yan T, Zhang A, Shi F, Chang F, Mei J, Liu Y and Zhu Y: Integrin $\alpha v \beta 3$-associated DAAM1 is essential for collagen-induced invadopodia extension and cell haptotaxis in breast cancer cells. J Biol Chem 293: 10172-10185, 2018.
11. Yu J, Chen L, Cui B, Widhopf GF II, Shen Z, Wu R, Zhang L, Zhang S, Briggs SP and Kipps TJ: Wnt5a induces ROR1/ROR2 heterooligomerization to enhance leukemia chemotaxis and proliferation. J Clin Invest 126: 585-598, 2016.

12. Zhang A, Yan T, Wang K, Huang Z and Liu J: PI3K $\alpha$ isoformdependent activation of RhoA regulates Wnt5a-induced osteosarcoma cell migration. Cancer Cell Int 17: 27, 2017.

13. Dai B, Yan $T$ and Zhang A: ROR2 receptor promotes the migration of osteosarcoma cells in response to Wnt5a. Cancer Cell Int 17: 112, 2017.

14. Xiong H, Yan T, Zhang W, Shi F, Jiang X, Wang X, Li S, Chen Y, Chen $\mathrm{C}$ and Zhu Y: miR-613 inhibits cell migration and invasion by downregulating Daam1 in triple-negative breast cancer. Cell Signal 44: 33-42, 2018.

15. Hasan MK, Yu J, Widhopf GF II, Rassenti LZ, Chen L, Shen Z, Briggs SP, Neuberg DS and Kipps TJ: Wnt5a induces ROR1 to recruit DOCK2 to activate Rac1/2 in chronic lymphocytic leukemia. Blood 132: 170-178, 2018.

16. Yu J, Chen L, Cui B, Wu C, Choi MY, Chen Y, Zhang L, Rassenti LZ, Widhopf Ii GF and Kipps TJ: Cirmtuzumab inhibits Wnt5a-induced Racl activation in chronic lymphocytic leukemia treated with ibrutinib. Leukemia 31: 1333-1339, 2017.

17. Yu J, Chen Y, Chen L, Zhang L, Rassenti LZ, Widhopf GF II and Kipps TJ: Cirmtuzumab inhibits ibrutinib-resistant, Wnt5a-induced Racl activation and proliferation in mantle cell lymphoma. Oncotarget 9: 24731-24736, 2018.

18. Choi MY, Widhopf GF II, Wu CC, Cui B, Lao F, Sadarangani A, Cavagnaro J, Prussak C, Carson DA, Jamieson C and Kipps TJ: Pre-clinical specificity and safety of UC-961, a first-in-class monoclonal antibody targeting ROR1. Clin Lymphoma Myeloma Leuk (15 Suppl): S167-S169, 2015.

19. Wu X, Yan T, Hao L and Zhu Y: Wnt5a induces ROR1 and ROR2 to activate RhoA in esophageal squamous cell carcinoma cells. Cancer Manag Res 11: 2803-2815, 2019.

20. Choi MY, Widhopf GF II, Ghia EM, Kidwell RL, Hasan MK, Yu J, Rassenti LZ, Chen L, Chen Y, Pittman E, et al: Phase I trial: Cirmtuzumab inhibits ROR1 signaling and stemness signatures in patients with chronic lymphocytic leukemia. Cell Stem Cell 22: 951.e3-959.e3, 2018.

21. Hasegawa D, Wada N, Yoshida S, Mitarai H, Arima M, Tomokiyo A, Hamano S, Sugii $\mathrm{H}$ and Maeda H: Wnt5a suppresses osteoblastic differentiation of human periodontal ligament stem cell-like cells via Ror2/JNK signaling. J Cell Physiol 233: 1752-1762, 2018.

22. Fritsch R and Downward J: SnapShot: Class I PI3K isoform signaling. Cell 154: 940-940.e1, 2013.

23. Linnerth-Petrik NM, Santry LA, Moorehead R, Jücker M, Wootton SK and Petrik J: Akt isoform specific effects in ovarian cancer progression. Oncotarget 7: 74820-74833, 2016.

24. Zhu Y, Shen T, Liu J, Zheng J, Zhang Y, Xu R, Sun C, Du J, Chen Y and Gu L: Rab35 is required for Wnt5a/Dvl2-induced Rac1 activation and cell migration in MCF-7 breast cancer cells. Cell Signal 25: 1075-1085, 2013.

25. Liu J, Zhang Y, Xu R, Du J, Hu Z, Yang L, Chen Y, Zhu Y and Gu L: PI3K/Akt-dependent phosphorylation of GSK3 $\beta$ and activation of RhoA regulate Wnt5a-induced gastric cancer cell migration. Cell Signal 25: 447-456, 2013. 